\title{
Thermal and dissipative effects in Casimir physics
}

\author{
M. Brown-Hayes,${ }^{1}$ J.H. Brownell, ${ }^{1}$ D.A.R. Dalvit,${ }^{2}$ W.-J. Kim,${ }^{1}$ A. Lambrecht,${ }^{3}$ F.C. Lombardo,${ }^{4}$ \\ F.D. Mazzitelli, ${ }^{4}$ S.M. Middleman, ${ }^{1}$ V.V. Nesvizhevsky,${ }^{5}$ R. Onofrio, ${ }^{1,6}$ and S. Reynaud ${ }^{3}$ \\ ${ }^{1}$ Department of Physics and Astronomy,Dartmouth College,6127 Wilder Laboratory, Hanover, NH 03755, USA \\ ${ }^{2}$ Theoretical Division, MS B213, Los Alamos National Laboratory, Los Alamos, NM 87545, USA \\ ${ }^{3}$ Laboratoire Kastler-Brossel, Université Pierre et Marie Curie, Campus Jussieu, F-75252 Paris, France \\ ${ }^{4}$ Departamento de Fisica J.J. Giambiagi, Facultad de Ciencias Exactas y Naturales, \\ Ciudad Universitaria, Pabellón 1, Universidad de Buenos Aires, 1428 Buenos Aires, Argentina \\ ${ }^{5}$ Institute Laue-Langevin, 6 rue Jules Horowitz, F-38042 Grenoble, France \\ ${ }^{6}$ Dipartimento di Fisica "G. Galilei", Università di Padova, Via Marzolo 8, Padova 35131, Italy
}

\begin{abstract}
We report on current efforts to detect the thermal and dissipative contributions to the Casimir force. For the thermal component, two experiments are in progress at Dartmouth and at the Institute Laue Langevin in Grenoble. The first experiment will seek to detect the Casimir force at the largest explorable distance using a cylinder-plane geometry which offers various advantages with respect to both sphere-plane and parallel plane geometries. In the second experiment, the Casimir force in the parallel plane configuration is measured with a dedicated torsional balance, up to $10 \mu \mathrm{m}$. Parallelism of large surfaces, critical for this configuration, is maintained through the use of inclinometer technology already implemented at Grenoble for the study of gravitationally bound states of ultracold neutrons. For the dissipative component of the Casimir force, we discuss detection techniques based upon the use of hyperfine spectroscopy of ultracold atoms and Rydberg atoms. Although quite challenging, this triad of experimental efforts, if successful, will give us a better knowledge of the interplay between quantum and thermal fluctuations of the electromagnetic field and of the nature of dissipation induced by motion of objects in quantum vacuum.
\end{abstract}

PACS numbers: 12.20.Fv, 42.50.Pq, 42.50.Lc, 04.80.Cc

\section{INTRODUCTION}

The study of quantum vacuum [1] is of great interest in physics due to recent astrophysical observations supporting an accelerating universe [2, 3, 4] . In various models, the acceleration is attributable to a cosmological term triggered by quantum vacuum effects [5]. In addition, the development of models unifying gravity and the other interactions has led to predictions of the existence of new Yukawian forces in the micrometer range with coupling of the same order of magnitude as gravity [ $[$ ]. These motivations are encouraging many theoretical and experimental studies into the physics of quantum vacuum and its interplay with cosmology and elementary particle physics [7, 8, 9, 10]. The Casimir force [1] is an important accessible window in this context (see [12, 13, 14, 15, 16, 17, 18, 19, 20] for monographs and reviews) and, particularly in the last decade, has been studied with an increasing level of accuracy in various geometries, from parallel plates [21, 22] to sphere-plane [23, 24, 25, 26, 27, 28, 29, 30, 31, 32]. Detailed knowledge of the Casimir force is important to master all the corrections in order to constrain, from the residuals of the actual experiments, the presence of Yukawian forces in the micrometer range. In particular, the interplay of Casimir forces with thermal photons due to a blackbody background and in presence of realistic cavities is not fully understood, and it seems likely that only experiments will be able to discern among the models proposed so far. The contribution of thermal photons grows with the size of the cavity in which the Casimir pressure is exerted, thus one needs to measure the Casimir force at large distances. Given the reduced sensitivity of atomic force microscope experiments at large distances and the weak signal expected with a sphere-plane geometry, the most promising configurations to look for Casimir forces at large distances are the cylinder-plane and the parallel plane geometries.

Thermal effects are not solely a result of finite temperature environment, since the motion of objects in quantum vacuum in itself also gives rise to heating. In fact, it has been predicted that non uniformly accelerated objects should dissipate energy in the form of photons, a phenomenon known as the dynamical Casimir effect [33, 34, 35, 36, 37, 38, 39, 40]. When specialized in a cavity configuration, this effect is also equivalent to parametric production of photons through quantum vacuum. The predicted Casimir photon production is quite low for realistic configurations, necessitating low-noise, high-sensitivity detection techniques such as hyperfine or Rydberg atomic spectroscopy. In this paper we discuss the current status of three ongoing experimental projects aimed at measuring the thermal contribution to the Casimir force and observing the predicted vacuum photons emitted from an non-uniformly accelerated resonant cavity. In section 2 we discuss the predictions for the thermal contribution to the Casimir force expected for both the cylinder-plane and the parallel plane configurations. We then describe some experimental issues for the corresponding experimental efforts ongoing at Dartmouth and in Grenoble, focusing on potential hurdles for the projects. Finally, in section 3, we briefly describe a proposal to detect the dynamical Casimir photons based upon atomic spectroscopy 

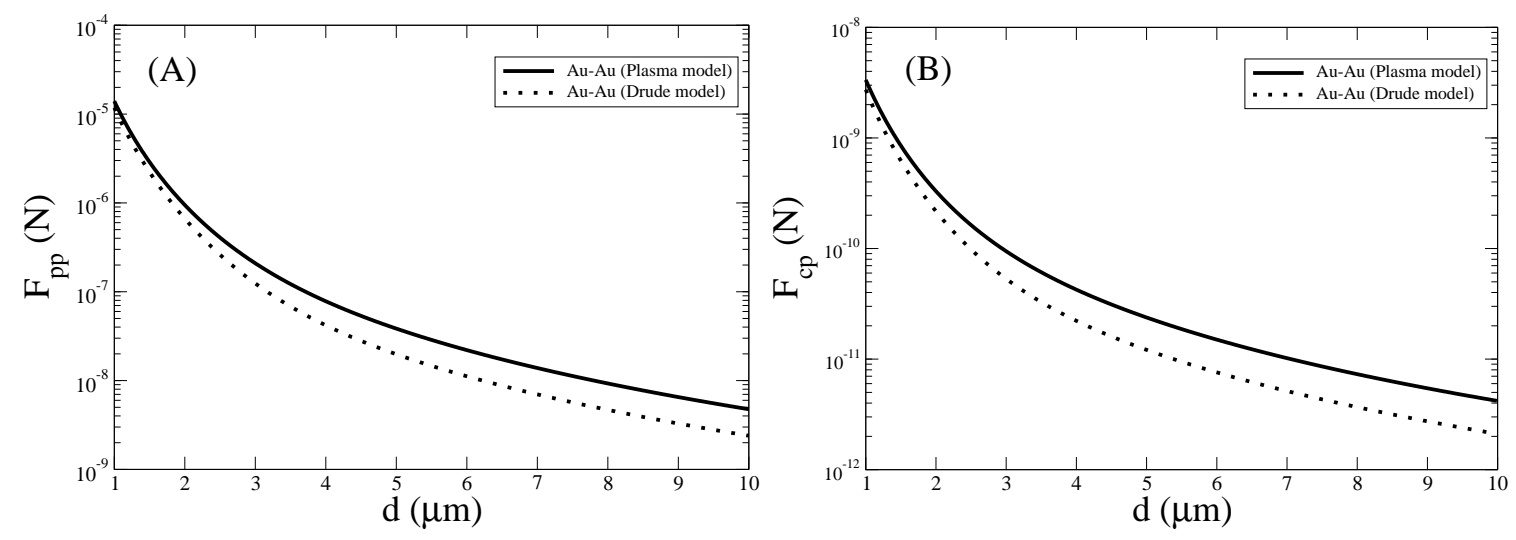

FIG. 1: Absolute Casimir force as a function of distance at $T=300 \mathrm{~K}$ for $\mathrm{Au}-\mathrm{Au}$ metallic surfaces in (A) plane-plane configuration and (B) cylinder-plane configuration. The force is computed using two distinct theoretical approaches: the plasma model (for which the TE $m=0$ mode contributes to the force), and the Drude model (for which the TE $m=0$ mode does not contribute). The plasma frequency of $\mathrm{Au}$ is $\omega_{p}=9.0 \mathrm{eV}$, and its relaxation frequency is $\nu=35 \mathrm{meV}$. For (A) we use $10 \mathrm{~cm} \times 12 \mathrm{~cm}$ plates. For (B) we use $L=2 \mathrm{~cm}$ for the length of the cylinder, and $a=1 \mathrm{~cm}$ for its radius. In the range of distances plotted in these figures, the Casimir force between $\mathrm{Cu}-\mathrm{Cu}$ and $\mathrm{Al}-\mathrm{Al}$ surfaces is the same as for $\mathrm{Au}-\mathrm{Au}$ surfaces within $0.1 \%$.

and high frequency mechanical resonators.

\section{THERMAL CONTRIBUTION TO THE CASIMIR FORCE}

The thermal contribution to the Casimir force has not yet been detected, in spite of its importance as a background to new forces in the $1 \div 10 \mu \mathrm{m}$ range. This scenario is complicated by the presence of models which yield different results when finite temperature and finite conductivity are taken into account [41, 42, 433, 44, 45, 46, 47, 48, 49, 50, 51, 52, 53, 54, 55, 56]. The basic theoretical formalism for the calculation of Casimir forces between real metals at finite temperature is given by the Lifshitz theory [57, 58]. This theory provides an expression for the pressure $P_{p p}$ (or, equivalently, the force $F_{p p}=S P_{p p}$ ) between two parallel plates of surface area $S$, separated by a gap $d$,

$$
F_{p p}(d)=\frac{S}{\pi \beta d^{3}} \sum_{m=0}^{\infty^{\prime}} \int_{m \gamma(d)}^{\infty} d y y^{2}\left[\frac{r_{\mathrm{TM}}^{-2} e^{-2 y}}{1-r_{\mathrm{TM}}^{-2} e^{-2 y}}+\frac{r_{\mathrm{TE}}^{-2} e^{-2 y}}{1-r_{\mathrm{TE}}^{-2} e^{-2 y}}\right]
$$

Here $\beta=1 / k_{\mathrm{B}} T$ is the inverse temperature, $\gamma(d)=2 \pi d / \beta \hbar c$, and the prime on the summation sign indicates that the $m=0$ term is counted with half weight. The reflection coefficients $r_{\mathrm{TE}}$ and $r_{\mathrm{TM}}$ for the two independent polarizations $\mathrm{TE}$ and TM are computed at imaginary frequencies $\omega_{m}=i \xi_{m}$, where $\xi_{m}=2 \pi m / \beta \hbar$ are the Matsubara frequencies.

The exact expressions for the reflectivity coefficients, which encompass the optical response of the electrons in the metallic surfaces, are not known. Within the Lifshitz formalism, they are expressed in terms of the dielectric permittivity $\epsilon(\omega)$ of the metals

$$
r_{\mathrm{TM}}^{-2}=\left[\frac{\epsilon\left(i \xi_{m}\right) p_{m}+s_{m}}{\epsilon\left(i \xi_{m}\right) p_{m}-s_{m}}\right]^{2} ; r_{\mathrm{TE}}^{-2}=\left[\frac{s_{m}+p_{m}}{s_{m}-p_{m}}\right]^{2}
$$

where $p_{m}=y / m \gamma$ and $s_{m}=\sqrt{\epsilon\left(i \xi_{m}\right)-1+p_{m}^{2}}$. The dielectric permittivity along the imaginary frequency axis can be computed using tabulated optical data for different metals. For the range of temperatures to be probed in the experiments (a few degrees around $T=300 \mathrm{~K}$ ), permittivity data corresponding to Matsubara frequencies $\zeta_{m}=2 \pi m / \beta \hbar$ with $m \geq 1$ can be extracted from the optical data. However, to determine the $m=0$ contribution, it is necessary to extrapolate the data to zero frequency. This extrapolation has been done by several groups using different approaches, leading to contradicting predictions for the magnitude of the force.

In Fig. 1 (left) we show the absolute value of the force between two parallel gold metallic plates at $T=300 \mathrm{~K}$ as a function of the gap using two different theoretical approaches. The first approach is based on the plasma model 
$\epsilon(i \xi)=1+\omega_{p}^{2} / \xi^{2}$, where $\omega_{p}$ is the plasma frequency. In this case, the TE $m=0$ term contributes to the Casimir force. The second approach is based on the Drude model $\epsilon(i \xi)=1+\omega_{p}^{2} /(\xi(\xi+\nu))$, where $\nu$ is the relaxation frequency. In this case the TE $m=0$ term does not contribute to the Casimir force. We have numerically computed the integral in Eq.(11) using quadratures with a cut-off $y_{\max }=50+m \gamma$, and we have used a cut-off $m_{\max }$ for the $m$ summation, corresponding to a maximum Matsubara frequency $\zeta_{\max }=10^{17} \mathrm{rad} / \mathrm{sec}$. We have checked that the results are robust against variations of these cut-offs. We also show in Fig. 1 (right) the force between a cylinder (of radius $a$ and length $L$ ) and a plane, assumed to be parallel, separated by a gap $d$. This force has been evaluated using the results for the pressure in the parallel plane configuration, and the proximity force approximation (valid in the limit $d \ll a$ ) $[59,60,61]$.

$$
F_{c p}=2 \int_{0}^{\pi / 2} P_{p p}(d+a(1-\cos \varphi)) L a d \varphi
$$

The angle $\varphi$ parameterizes the location of the infinitesimal surfaces on the cylinder. The leading contribution to the integral comes from angles close to $\varphi=0$. In order to speed up the numerical computation of the integral, we have used as an upper limit $\varphi=\pi / 20$, and we have checked that the results, in the limit $d \ll a$, are robust when we varied this upper limit.

In the proximity force approximation the surfaces of the plane and the cylinder are divided into infinitesimal parts to integrate the parallel plates result. There is an ambiguity in the choice of the areas of these infinitesimal parts. Different choices give distinct approximations for the force. In Eq. 3 above, we have chosen the area of a small portion of the cylinder $d A_{c}=\operatorname{Lad} \varphi$. One could also use the area of a small portion of plane $d A_{p}=L a \cos \varphi d \varphi$, or a combination of the two, like the geometric mean $d A_{g m}=\left(d A_{p} d A_{c}\right)^{1 / 2}$. The accuracy of the proximity force approximation has been estimated by computing the force $F_{c p}$ using all the areas mentioned above. The results differ by less than $1.2 \%$ for both plasma and Drude models at $d<10 \mu \mathrm{m}$.

Analytical expressions for the force, both for parallel plates and cylinder-plate geometries, can be obtained for some limits. We consider here the case of surfaces without roughness (corrections due to roughness are important for small distances $d<1 \mu \mathrm{m}$ at $T=300 \mathrm{~K}$ ). For perfect conductors at zero temperature one recovers the original Casimir formula for the parallel plates case, and the cylinder-plane force can be obtained in the proximity force approximation $(d \ll a)[62]$

$$
F_{p p}^{(T=0)}=\frac{\pi^{2} \hbar c}{240} \frac{S}{d^{4}} ; F_{c p}^{(T=0)}=\frac{\pi^{3} \hbar c L a^{1 / 2}}{384 \sqrt{2} d^{7 / 2}}
$$

The scaling of the force with distance for the cylinder-plane case $\left(\simeq d^{-7 / 2}\right)$ is intermediate between the parallel plane $\left(\simeq d^{-4}\right)$ and the sphere-plane $\left(\simeq d^{-3}\right)$ cases. This is an advantage with respect to the parallel plane case because the latter is quickly going to zero with the distance. For reasonable values of the relevant parameters, the magnitude of the Casimir force in the cylinder-plane case is also intermediate between the cases of the sphere-plane, as investigated with atomic force microscopy, and the case of parallel planes [63]. For finite temperature and real conductors, one can obtain closed expressions for the force using, e.g., the plasma model. At temperatures $T \ll T_{\text {eff }}=\hbar c / 2 k_{\mathrm{B}} d$, and to first order in $\delta / d$ (where $\delta=\lambda_{p} / 2 \pi$, with $\lambda_{p}$ the plasma wavelength) one obtains

$$
\begin{aligned}
& \frac{F_{p p}}{F_{p p}^{(T=0)}} \approx 1+\frac{1}{3}\left(\frac{T}{T_{\mathrm{eff}}}\right)^{4}-\frac{16 \delta}{3 d}\left[1-\frac{45 \xi(3)}{8 \pi^{3}}\left(\frac{T}{T_{\mathrm{eff}}}\right)^{3}\right] \\
& \frac{F_{c p}}{F_{c p}^{(T=0)}} \approx 1+\frac{132.096 \zeta(7 / 2)}{\pi^{9 / 2}}\left(\frac{T}{T_{\mathrm{eff}}}\right)^{7 / 2}-\frac{\delta}{d}\left[\frac{14}{3}-\frac{48 \xi(3)}{\pi^{3}}\left(\frac{T}{T_{\mathrm{eff}}}\right)^{3}\right],
\end{aligned}
$$

where $\xi(z)$ is the Riemann zeta function. Finally, in the high temperature limit $T \gg T_{\text {eff }}$ the forces are dominated by the thermal contribution

$$
F_{p p}^{\text {thermal }} \approx \frac{\xi(3) k_{\mathrm{B}} T}{4 \pi} \frac{S}{d^{3}} ; F_{c p}^{\text {thermal }} \approx \frac{3 \xi(3) k_{\mathrm{B}} T}{16 \sqrt{2}} \frac{L a^{1 / 2}}{d^{5 / 2}},
$$

which represent the radiation pressure due to the thermal blackbody photons at finite temperature. This force could be used, by intentionally increasing the temperature by known amounts, to obtain physical calibrations and estimates of the sensitivity for any apparatus aimed at high-precision measurements of the Casimir force. 


\section{A. Cylinder-plane configuration: experimental issues}

The apparatus we are developing to measure the Casimir force in a cylinder-plane configuration has been described in 65] as are parallelization and calibration techniques. Here we focus on possible complications in measuring the Casimir force due to the finite thermal stabilization of the apparatus. This is reflected first of all in the stability of the parallelism. Long term thermal drifts on the apparatus affect the parallelism and therefore limit the total measurement time. As discussed in [65], the parallelism can be assessed by intentionally rotating the cylinder around its midpoint. The parallel configuration is that which minimizes the frequency-shift due to an external spatially dependent force, such as the electrostatic force, and this is shown in Fig. 2 for a relatively long measurement time. The curves are fitted as pieces of parabolas but both the curvature and the center of the parabolas are different, with the shallower curve corresponding to a larger distance between the cylinder and the plane. This can be interpreted as due to sudden drifts in the separation distance, and will be mitigated in future versions of the apparatus with improved thermal stabilization and the use of materials like Invar alloys with minimal thermal expansion coefficients.

The thermal drifts are also an indirect reason for limiting the number of averages in a long-time measurement of the Casimir force. A measurement of the Casimir force around $3 \mu \mathrm{m}$ capable of disentagling between the two different models for the temperature corrections, as discussed above and in [65], requires high force sensitivity. One possible detection scheme, which has been used to calibrate the apparatus, is based on the measurement of frequency shifts of the resonator induced by a spatially-dependent force [66, 67] In the (ideal) case of a perfect stability (no drifts in frequency or other parameters), an ideal theoretical understanding of the response, and an infinite number of measurements, one could achieve an exact determination of the peak frequency for any value of the mechanical quality factor. Obviously, none of these conditions are fulfilled in an actual experiment. The parameters of the resonator may drift in time, there may be an incomplete understanding of the background which produces residuals in the fit, as well as statistical fluctuations due to the finite number of averages. This gives then rise to a finite precision in determining any parameter modeling the resonator response, including the peak frequency. We have studied the dependence of the precision in the determination of the peak frequency versus the number of averages of the FFT transform of the photodiode signal resulting from a fiber optic interferometer [68]. As shown in Fig. 3 (left) the error in the determination of the peak frequency is fitted by the standard $1 / \sqrt{N}$ dependence. Due to the presence of thermal drifts, one cannot expect ideal behaviour even in the limit of infinite averaging. It is therefore important to study how to optimize the precision in the determination of the peak frequency for a fixed measurement time. Once the latter is fixed we can choose to measure on a large window (at the price of worse frequency resolution on the FFT analysis) or viceversa. In Fig. 3 (right) we plot the error in the determination of the peak frequency versus the selected window

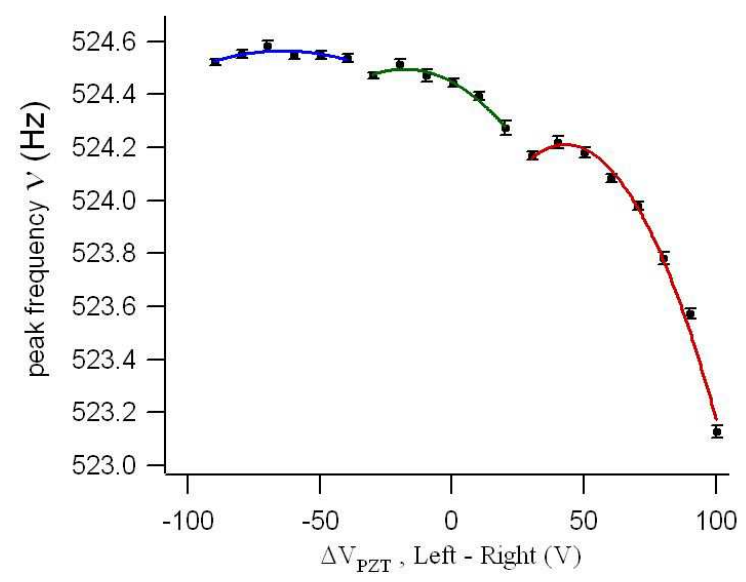

FIG. 2: Long-term behaviour of the resonator frequency. Depicted is the frequency of the resonator with an applied bias voltage of $V=100 \mathrm{~V}$ versus the difference between the voltages applied to the piezoelectric actuators, starting from the highest voltage $\mathrm{V}_{\mathrm{L}}-\mathrm{V}_{\mathrm{R}}=+100 \mathrm{~V}$ and scaling down in time (from right to left) to minimize hysteresis effects in the piezoelectric actuators. The free resonator frequency (without bias voltage) is $\nu_{0}=(526.40 \pm 0.02) \mathrm{Hz}$. Each measurement carries 100 averages (each average lasting $32 \mathrm{~s}$ ), and after the first 7 measurements we experienced an abrupt change in distance (manifested both by a swallower curvature and a higher frequency) as well as in the angle formed by the cylinder axis and the resonator surface (manifested by a displacement in the voltage for which there is the minimum frequency shift). A second abrupt change has taken place after another 6 measurements, in the same direction for the drift of both the gap distance and angle. The continuous curves are the distinct parabolic fits to each set of data. The overall duration of the run was $\simeq 18$ hours. 

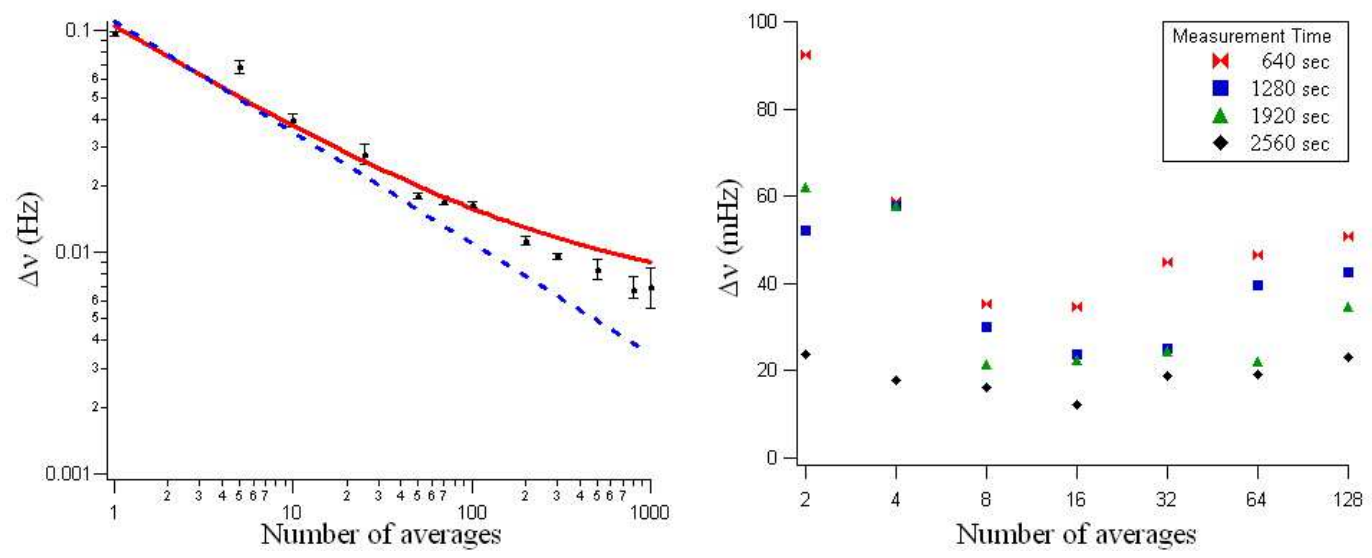

FIG. 3: Optimization of the accuracy for the peak frequency measurement. On the left panel we plot the peak frequency uncertainty evaluated from the fitting of the resonance curve of the mechanical oscillator versus the number of averages taken with the FFT spectrum analyzer at constant sampling time (32 s, corresponding to a frequency span of $12.5 \mathrm{~Hz})$. The data are fitted with a $1 / \sqrt{N}$ dependence (dashed line), as well as with a constant offset term $\Delta \nu=a+b \sqrt{N}$ (continuous line). The second fit gives us an offset term $a=(5.9 \pm 2.4) \mathrm{mHz}$ which is related to the systematics in data fitting and the stability of the fit parameters and the apparatus. On the right panel we report the error on the resonance curve fitting versus the number of averages, keeping constant the total measurement time in each of the four data sets, corresponding to 640 sec and its three next multiples. Each average corresponds to different FFT windows (spanning from $200 \mathrm{~Hz}$ in the case of $2 \mathrm{~s}$ sampling time to $3.125 \mathrm{~Hz}$ for $128 \mathrm{~s}$ sampling time) and then different FFT frequency resolutions. Consistent with the plot on the left side, the peak frequency error on the right plot decreases from one data set to the other corresponding to increasing measurement time. The four curves also show a common trend with a minimization of the error for a number of averages between 8 and 16 , corresponding to a frequency window $\delta \nu$ between 25 and $50 \mathrm{~Hz}$, i.e. about 10 times the resonator linewidth of $\simeq 3 \mathrm{~Hz}$. Error bars represent the statistical uncertainty of the fitting procedure.

of the FFT analyzer (or, equivalently, versus the sampling time for each measurement). It is evident that the error on the peak frequency is optimized for an intermediate value of the windowing. Larger windowing yields poor frequency resolution inherent to the FFT, partially compensated by the decreased statistical uncertainty resulting from the larger number of averages, while small windowing allows for better frequency resolution at the cost of large statistical fluctuations. Our tests indicate that the error on the peak frequency is minimized by using a window about ten times the intrinsic bandwidth of the mechanical resonator (on the order of $2-3 \mathrm{~Hz}$ in our case). This also shows that, unless particular care is taken to minimize thermal drifts, the use of large mechanical quality factors for the resonators does not necessarily improve the sensitivity, as the longer measurement times required for the optimal narrower windowing will make the resonator more prone to thermal drifts: in the presence of finite thermal stability there will be, for the frequency-shift measurement technique, an optimal quality factor for the resonator.

\section{B. Parallel plane configuration: experimental issues}

The parallel plate configuration, as discussed in [69], has still a high priority for performing Casimir force measurements due to the unique features of this geometry. In particular, it may provide the strongest limits on non-Newtonian gravity in the 1-10 $\mu \mathrm{m}$ range due to the largest attainable signal and the absence of gravitational force gradients apart from boundary effects [70, 71, 72]. Due to the importance of investigating non-Newtonian forces in recent unification frameworks, this research program is currently pursued by our collaboration. In order to measure the thermal contribution, the steep scaling of the parallel plane Casimir force with respect to distance requires the use of large, macroscopic surfaces, which in turn leads to the use of a high-sensitivity torsional balance [73]. In our project, two pairs of plates are installed on opposite arms of the moving and static parts of the balance, respectively (see Fig. 4).

These plates have a surface area of $120 \mathrm{~cm}^{2}$ for the measurement at the largest gaps, on the order of $10 \mu \mathrm{m}$. For smaller distances $(\simeq 1 \mu \mathrm{m})$ the effective area can be reduced to $15 \mathrm{~cm}^{2}$, which minimizes the chance of having dust trapped in the gap. The target torque sensitivity is in the 1-100 $\mu \mathrm{N} / \mathrm{rad}$ range, obtainable with quartz wire of diameter 50-150 $\mu \mathrm{m}$. This allows for a minimum detectable force of order $1 \mathrm{pN}$. Three high precision piezoelectric actuators and their feedback controllers maintain a constant distance between the two plates throughout the duration of the measurement, with an accuracy of $0.2 \mu \mathrm{m}$. Capacitors symmetrically located on the opposite sides of the 
active surfaces for the measurement of the Casimir force allow for further control of the gap distance. The distance between the capacitor plates is large enough $(100 \mu \mathrm{m})$ to avoid backaction effects on the torsional balance, yet provide adequate sensitivity.

Parallelization procedures will be based on expertise already acquired in Grenoble through an experiment in which discretization of the energy levels of ultracold neutrons in the Earth gravitational field has been observed [74]. While in these experiments parallelism between the neutron reflecting surface and the absorber has been kept to about $10^{-6}$ radians, the expected parallelism with the use of the piezoelectric actuators and auxiliary capacitors is around $10^{-7}$ radians. The surface roughness should be around 1-2 nm. In a second stage of the experiment, metallic layers with thickness of $\simeq 1 \mu \mathrm{m}$ will be used when searching for hypothetical Yukawa forces with gravitational coupling, and this may lead to a degradation of the surface quality. The availability of in situ diagnostic techniques in surface science laboratories already present in Grenoble will provide complete characterization of the surfaces.

Another potential hurdle along the way is the tilting of the mirrors in the arms of the torsional balance. This can be addressed by reducing the lever of the torsional balance, properly designing the position of its pivot point, and segmenting the electrodes in such a way that small tilts do not result in changes of the effective surface. The latter solution also implies a control of the border effects, which have to be carefully estimated for instance through the worldline numerics approach pioneered in [75]. The experiment is currently in preparation (see Figs. 5) and the first calibrations of the apparatus are planned in early 2006. Once again, we want to stress that various physical signals can be used to calibrate this apparatus, including the possibility of measuring the gravitational force through a Cavendish-like experiment. This allows for a simple expression of the apparatus sensitivity in terms of the maximum distance at which a known gravitational source may be detected - a parametrization of the sensitivity particularly natural when discussing the limits to Yukawa forces in the micrometer range.

\section{DISSIPATIVE CONTRIBUTION TO THE CASIMIR FORCE}

The emission of photons due to the dissipative nature of motion in quantum vacuum has been predicted using various analytical [33, 34, 37, 38, 39, 40] and numerical [76]. Dissipation is expected when a material body undergoes a non uniform acceleration. The phenomenon is often discussed in the context of a resonant cavity where the amplification of vacuum field energy is enhanced due to a periodic motion of a boundary and the build-up of photons in the cavity. From this point of view the phenomenon, also called dynamical Casimir effect, consists of the amplification of vacuum field energy as the periodic modulation of the cavity boundaries excites a particular mode of vacuum field, with the transformed state being squeezed. The number of photons in the mode (if uncoupled to any other mode) increases exponentially as $N_{\text {cas }}=\sinh ^{2}(\lambda \epsilon t)$, where $\epsilon=\delta L / L$ is the amplitude of the modulation and $\lambda$ depends on the geometry of the cavity and on the particular resonant mode [77]. In practice the exponential growth is constrained

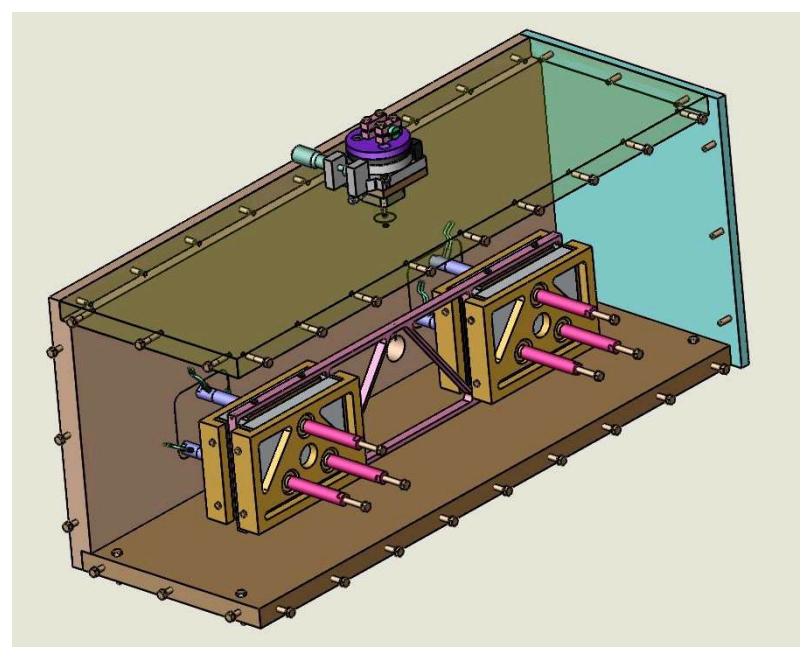

FIG. 4: Schematic design of the torsional balance under development at ILL, Grenoble. The parallelism between the plates of the balance and the outer plates is obtained through a set of high-precision piezoelectric actuators. The symmetry of the scheme allows for many cross-checks of systematic effects, and for physical calibrations and assessments of the ultimate sensitivity based on both electrostatic and gravitational forces, the latter force being testable through fine control of the position of nearby static masses. 

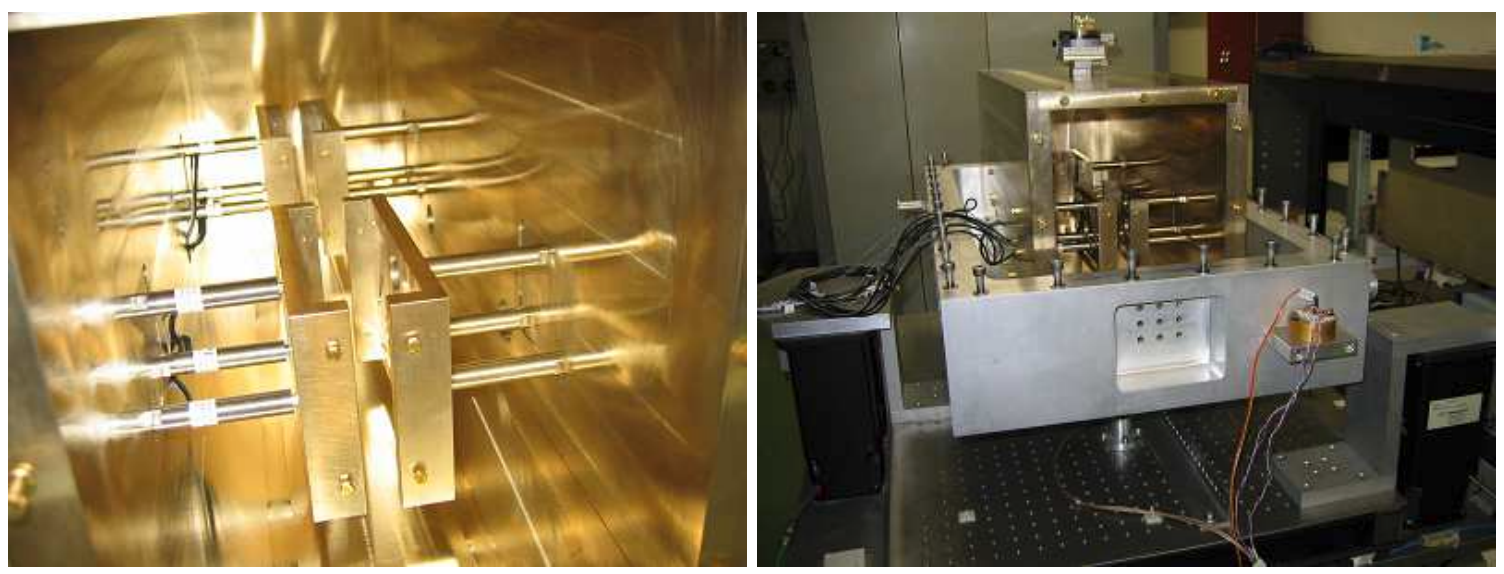

FIG. 5: Experimental set-up for the measurement of the Casimir force in a parallel plane configuration. On the left, the plate holders are connected to the external frame through three precision pieoelectric positioners each plate. On the right is an overall view of the vacuum chamber with the fiber holder on the top.

due to the finite optical quality factor $Q$, with the growth saturating at times $\tau_{\text {sat }} \simeq Q / \omega$. Typically, for cylindrical cavities with rectangular or circular sections, $\lambda / \omega \sim \mathcal{O}(1)$. Therefore the number of produced photons is approximated as $N_{\text {cas }} \sim \sinh ^{2}(Q \epsilon)$. For $\epsilon \simeq 10^{-8}$ (about a few nm displacement in the GHz range) and $Q \simeq 10^{8}$, a typical number of photons expected is on order of unity. The result is very sensitive to the value of $Q \epsilon$, the photon number being $\simeq 10^{3}$ for $Q \epsilon \sim 4$. These estimations illustrate the difficulty of the observation of this phenomenon. The number of created photons could be much larger if, instead of considering a cavity with moving boundaries, one considers a cavity with time-dependent reflectivity. The effective length of the cavity could be changed by irradiating with fast laser pulses a thin semiconductor film contained in it [78, 79, 80]. It has been recently shown, using a simplified model [81], that in this case one could reasonably get $Q \epsilon \gg 1$, but the heating due to the laser pulses and the generation of excitations in the semiconductor [82] are an open issue for the current implementation of this scheme as in the MIR experiment [83, 84, 85]. Recently, a generation and detection scheme has been proposed based on the two emerging technologies of high-frequency resonators and the ultracold atoms [69]. The basic idea is to prepare two-level atoms into a particular excited state and to induce the stimulated transition in the presence of the amplified vacuum field as schematically depicted in Fig. 6 .

The vacuum amplification takes place inside a resonant cavity where an array of mechanical resonators are coherently oscillating. These mechanical resonators of high frequency are available (up to $3.1 \mathrm{GHz}$ ) due to the recent development of the Film Bulk Acoustic Resonator (FBAR) devices. The integral part of the FBAR devices is a thin piezoelectric film made of Aluminum Nitride (AIN) for which the mechanical frequency can be changed with a varying thickness [86, 87]. Depending on the nature of two-level atoms, one could detect the progressive change in atomic population either by fluorescence (photon detection) or by field ionization procedure (electron detection). The original scheme relies on the hyperfine splitting of ${ }^{6} \mathrm{Li}$ whose transition frequency in the ground state is $228 \mathrm{MHz}$, which is the lowest among alkali atoms, thereby allowing a reasonable matching with the working frequency of available high-
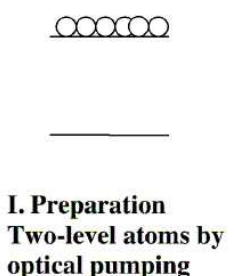

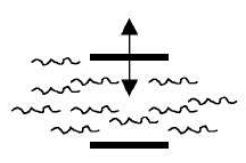

$$
\begin{aligned}
& \text { II. Generation } \\
& \text { Mechanical vibration } \\
& \text { of a resonant cavity }
\end{aligned}
$$
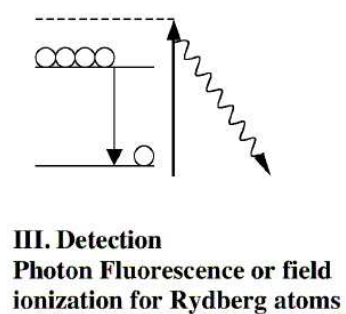

FIG. 6: Schematic of the atomic detection technique. The two-level atoms interact with the photons generated through the mechanical modulation of the cavity. In the detection stage, either fluorescence or field ionization techniques can be adapted to detect the change in the atomic population. 
frequency mechanical nanoresonators. Apart from the difficulty of fabricating an ensemble of mechanical resonators coherently driven at a well-defined frequency $(456 \mathrm{MHz})$, the use of hyperfine splitting seems disadvantageous due to an extremely low stimulated emission rate. For a two-level atom, the stimulated emission rate is directly proportional to the spontaneous rate given by

$$
A=\frac{\omega^{3} D_{i j}^{2}}{\pi \epsilon_{o} \hbar c^{3}}
$$

where $D_{i j}^{2}$ is the matrix element for the dipole transition between $i$ th and $j$ th states. For the two hyperfine ground states of Li, the magnetic dipole interaction dominates the transition, which gives rise to an additional factor of $v^{2} / c^{2}$,

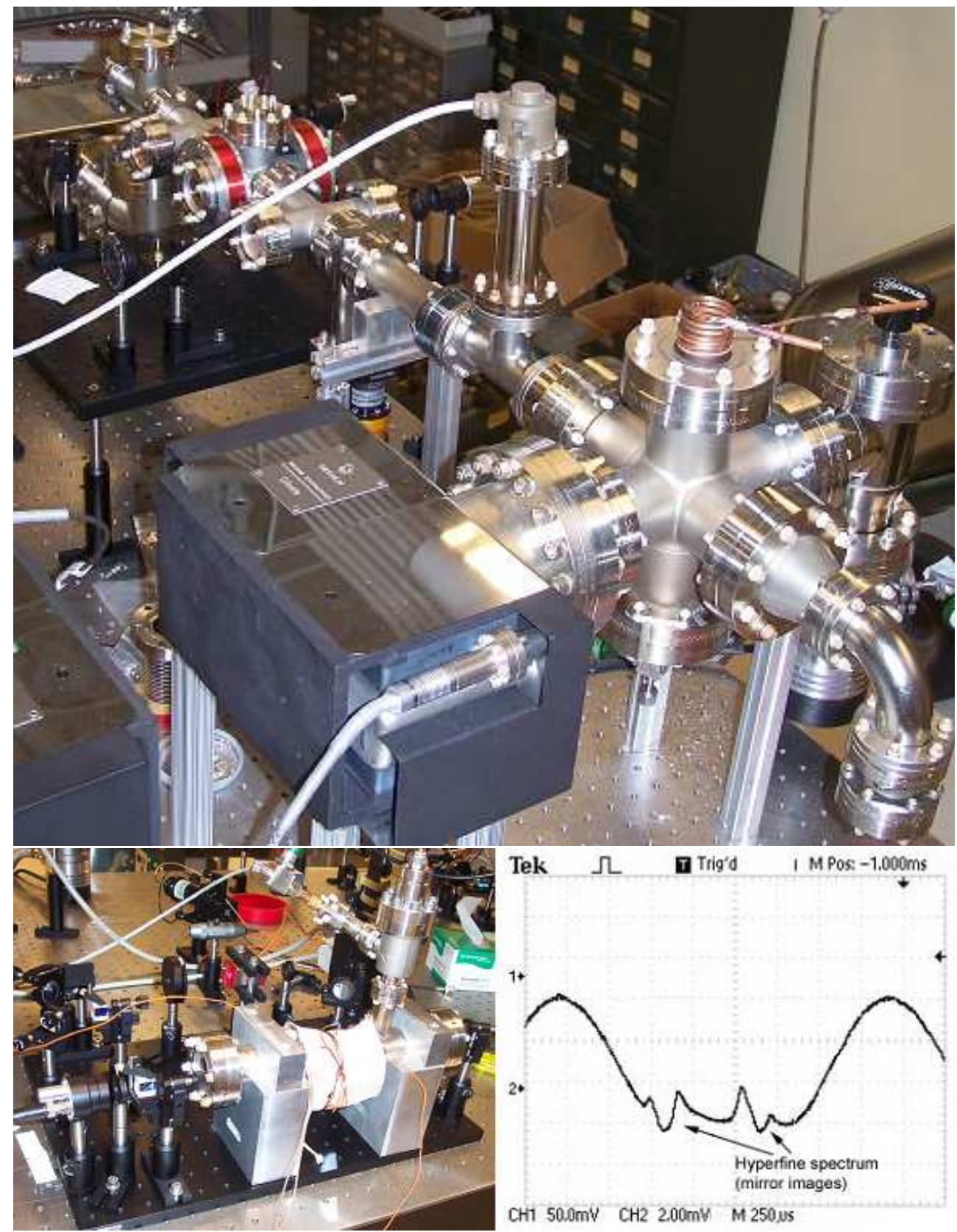

FIG. 7: Atomic beam line for studies of the induced transitions in hyperfine levels of ${ }^{6}$ Li by means of controllable electromagnetic fields (top picture). ${ }^{6} \mathrm{Li}$ vapor cell (bottom left) and corresponding saturation absorption signal with the hyperfine states spaced by $228 \mathrm{MHz}$ (bottom right). 
lowering the emission rate by $10^{20}$ compared to the usual electric dipole interaction. Furthermore, the estimated power of Casimir photons for $Q \epsilon \sim 1$ is $N_{\text {cas }} \hbar \omega / \tau_{\text {sat }} \simeq 10^{-25} \mathrm{~W}$, too small to induce the hyperfine transition.

In order to maximize the atomic emission rate, one could consider an electric dipole transition with a larger matrix element. The most natural choice satisfying these criteria is the use of circular Rydberg atoms [88, 89] having large electric dipole moment $\left(\simeq n^{4} a^{2}\right)$ and relatively long lifetime $\left(\simeq n^{5}\right)$ 90, 91], already employed in the studies of cavity quantum electrodynamics [92, 93] and quantum information processing [94]. The transition frequency can in principle be chosen at will by varying the principal quantum number up to $n=150$ [95, 96], which provides a great tunability with a mechanical resonator.

Although the use of Rydberg atoms greatly increases the stimulated emission rate, the number of photons generated inside the cavity is still too small to be measured through an appreciable change in atomic population. This issue can be overcome by exploiting an additional amplification procedure through super-radiance. The overall scheme is identical to the super-radiant maser system in the millimeter wave domain considered in [97, 98]. In super-radiance a single atom undergoing a transition triggers a subsequent emission on the neighboring atoms resulting in a large pulse whose lifetime is inversely proportional to the number of atoms present in the coherent volume. As long as the number of Casimir photons produced inside a resonant cavity exceeds the number of spontaneously emitted photons during the atomic travel time across the cavity, it is possible to measure the triggering effect of the Casimir photons on the super-radiant pulse through control of delay time, polarization, and phase [98]. Because the estimated number of Casimir photons is very small however, the triggering effect may be dominated by the vacuum fluctuations or the presence of thermal photons as well. For this super-radiance scheme to be effective, the Casimir photons must be the dominant triggering source. So far, we have built at Dartmouth a ${ }^{6} \mathrm{Li}$ atomic beam source to study optical pumping and detection of hyperfine states populations (see Fig. 7), which can be easily extended to rubidium atoms, the most natural candidate for the preparation of Rydberg states in our scheme.

\section{CONCLUSIONS}

We have described ongoing experimental efforts in Casimir physics, with the goal of studying the interplay between pure quantum fluctuations and thermal effects, either in the form of thermal photons - always present in any finite temperature environment, or photons originating from the dissipative nature of non-uniformly accelerated motion of an object in quantum vacuum. The combination of strong signals at large distances and relatively simple parallelization makes the investigation of the Casimir force in a cylinder-plane geometry quite attractive, allowing for discrimination between theoretical approaches. Recent technological advances make possible the achievement of almost ideal parallelization in the parallel plane geometry, providing strong constraints on hypothetical Yukawian forces. Use of hyperfine or super-radiant Rydberg atoms for the detection of the dynamical Casimir effect will require a delicate balance in the parameter space, and yet it promises exciting insights into the dissipative nature of quantum vacuum. These combined efforts should lead towards a more comprehensive understanding of fluctuation and dissipation of quantum vacuum.

\section{Acknowledgments}

This paper is based upon a plenary talk by R. Onofrio and a poster by W.J. Kim, who are grateful to the organizers of QFEXT'05 for the kind invitation and partial financial support, respectively. M. Brown-Hayes acknowledges support from the Dartmouth Graduate Fellowship, W. J. Kim acknowledges support from Gordon Hull Fellowship program, and S.M. Middleman acknowledges support through an undergraduate Mellam fellowship. F. C. Lombardo and F. D. Mazzitelli acknowledge support from Universidad de Buenos Aires, CONICET and ANPCyT. We thank N. Monnig for experimental assistance, and R. L. Johnson for skillful technical support. We are grateful to M. Antezza, H. Gies, P. Milonni, V. Mostepanenko, L. Pitaevskii, and G. Ruoso for fruitful discussions.

[1] Milonni P 1994 The Quantum Vacuum (San Diego: Academic Press)

[2] Riess AG et al. 1998 Astron. J. 1161009

[3] Garnavich PM et al. 1998 Astrophys. J. 50974

[4] Perlmutter S et al. 1999 Astrophys. J. 517565

[5] Peebles PJE and Rata B 2003 Rev. Mod. Phys. 75559

[6] Fischbach E and Talmadge C L 1999 The Search for Non-Newtonian Gravity (New York : AIP/Springer-Verlag, New York) 
[7] Zeldovich Y B 1967 Sov. Phys. JETP 6316

[8] Weinberg S 1989 Rev. Mod. Phys. 611

[9] Carroll S M 2001 Living Rev. Rel. 41

[10] Sahni V and Starobinsky A A 2000 Int. J. Mod. Phys. D 9373

[11] Casimir H B G 1948 Proc. K. Ned. Akad. Wet. B 51793

[12] Plunien G, Müller B, and Greiner W 1986 Phys. Rep. 13487

[13] Elizalde E and Romeo A 1991 Am. J. Phys. 59711

[14] Mostepanenko V M and Trunov N N 1997 The Casimir Effect and its Applications (London: Clarendon)

[15] Bordag M 1999 The Casimir Effect 50 Years Later (Singapore: World Scientific)

[16] Bordag M, Mohideen U, and Mostepanenko V M 2001 Phys. Rep. 3531

[17] Reynaud S et al. 2001 C. R. Acad. Sci. Paris IV-2 1287

[18] Milton K A 2001 The Casimir Effect: Physical Manifestations of the Zero-Point Energy (Singapore: World Scientific)

[19] Milton K A 2004 J. Phys. A: Math. Gen. 37 R209

[20] Lamoreaux S K 2005 Rep. Prog. Phys. 68201

[21] Sparnaay M J 1958 Physica 24751

[22] Bressi G, Carugno G, Onofrio R, and Ruoso G 2002 Phys. Rev. Lett. 88041804

[23] van Blokland P H G M and Oveerbeek J T G 1978 J. Chem. Soc. Faraday Trans. I 742637

[24] Lamoreaux S K 1997 Phys. Rev. Lett. 785

[25] Mohideen U and Roy A 1998 Phys. Rev. Lett. 814549

[26] Harris B W, Chen F, and Mohideen U 2000 Phys. Rev. A 62052109

[27] Chan H B, Aksyuk V A, Kleiman R N , Bishop D J, and Capasso F 2001 Science 2911941

[28] Chan H B et al. 2001 Phys. Rev. Lett. 87211801

[29] Decca R S, Lopez D, Fischbach E, and Krause D E 2003 Phys. Rev. Lett. 91050402

[30] Iannuzzi D, Gelfand I, Lisanti M, and Capasso F 2004 Proc. Nat. Ac. Sci. USA 1014019

[31] Decca R S et al. 2005 Phys. Rev. Lett. 94240401

[32] Decca R S et al. 2005 Annals of Physics 31837

[33] Moore G T 1970 J. Math. Phys. 112679

[34] Law C K 1991 Phys. Rev. Lett. 731931

[35] Schwinger J 1993 Proc. Natl. Acad. Sci. U.S.A. 90958

[36] Cole C K and Schieve W C 1995 Phys. Rev. A 524405

[37] Lambrecht A, Jaekel M T, and Reynaud S 1996 Phys. Rev. Lett 77615

[38] Plunien G, Schützhold R, Soff G 2000 Phys. Rev. Lett. 841992

[39] Dodonov V V 2001 Adv. Chem. Phys. 119309

[40] Crocce M, Dalvit D A R, Mazzitelli F D 2002 Phys. Rev. A 66033811

[41] Lambrecht A and Reynaud S 2000 Eur. Phys. J. D 8309

[42] Bostrom M and Sernelius B E 2000 Phys. Rev. Lett. 844757

[43] Lamoreaux S K 2001 Phys. Rev. Lett. 87139101

[44] Sernelius B E 2001 Phys. Rev. Lett. 87139102

[45] Chen F, Klimchitskaya G L, Mohideen U, and Mostepanenko V M 2003 Phys. Rev. Lett. 90160404

[46] Geyer B, Klimchitskaya G L, and V.M. Mostepanenko V M 2003 Phys. Rev. A 67062102

[47] Geyer B, Klimchitskaya G L, and V.M. Mostepanenko V M 2002 Phys Rev A 65062109

[48] Esquivel R, Villarreal C, and Mochan W L 2003 Phys. Rev. A 68521031

[49] Genet C, Lambrecht A, and S. Reynaud S 2002 Int. J. Mod. Phys. A 17761

[50] Genet C, Lambrecht A, and Reynaud S 2000 Phys. Rev. A 62012110

[51] Svetovoy V B and Lokhanin M V 2003 Phys. Rev. A 67022113

[52] Torgerson J R and Lamoreaux S K 2004 Phys. Rev.E $\mathbf{7 0} 047102$

[53] Esquivel R and Svetovoy V B 2004 Phys. Rev. A 69062102

[54] Bordag M, Geyer B, Klimchitskaya G L, and Mostepanenko V M 2000, Phys. Rev. Lett. 85503

[55] Høye J S et al. 2003 Phys. Rev. E 67056116

[56] Brevik I et al. 2005 Phys. Rev. E 71056101 V. S. Bentsen et al., quant-ph/0505136

[57] Lifshitz E M 1956 Zh. Eksp. Teor. Fiz 2994 [Sov. Phys. JETP 2, 73 (1956)]

[58] Lifshitz E M and Pitaevskii L P 2002 Statistical Physics, Part 2 (Oxford: Butterworth-Heinemann)

[59] Derjaguin B V and Abrikosova I I 1957 Sov. Phys. JETP 3819

[60] Derjaguin B V 1960 Sci. Am. 20347

[61] Blocki J, Randrup J, Swiatecki W J and Tsang F 1977Ann. Phys. 105427

[62] Dalvit D A R, Lombardo F C, Mazzitelli F D, and Onofrio R. 2004 Europhys. Lett. 67517

[63] The unique case of sphere-plane experiment using a lens as the spherical surface gives a large signal 24], however the precision in the measurement of the radius of curvature is an issue [64]

[64] Lamoreaux S K 1998 Phys. Rev. Lett. 815475

[65] Brown-Hayes M, Dalvit D A R, Mazzitelli F D, Kim W J, and Onofrio R 2005 Phys. Rev. A 72051102

[66] Giessbl F J 2003 Rev. Mod. Phys. 75949

[67] Bressi G, Carugno G, Galvani A, Onofrio R, Ruoso G, and Veronese F 2001 Class. Quantum Grav. 183943

[68] Rugar D, Marmin H J, and Guethner P 19891989 Appl. Phys. Lett. 552588

[69] Onofrio R 2004, in Proceedings of the Sixth International Workshop on Quantum Fields Theory under the Influence of 
External Conditions, K. A. Milton editor (Princeton: Rinton)

[70] Price J C 1988, in Proceedings of the International Symposium on Experimental Gravitational Physics, P. F. Michelson editor (Singapore, World Scientific)

[71] Onofrio R and Carugno G 1995 Phys. Lett. A 198365

[72] Carugno G, Fontana Z, Onofrio R, and Rizzo C 1997 Phys. Rev. D 556591

[73] Lambrecht A, Nesvizhevsky V V, Onofrio R, and Reynaud S 2005 Class. Quantum Grav. 225397

[74] Nesvizhevsky V V et al. 2003 Phys. Rev. D 67102002

[75] Gies H, Langfeld K, and Moyaerts L 2003 J. High Energy Phys. 618

[76] Ruser M 2005 J. Opt. B7 S100

[77] Crocce M, Dalvit D A R, Lombardo F C, and Mazzitelli F D, 2005, J. Opt. B7 S32

[78] Lozovik Y E, Tsvetus V G, and Vinogradov E A 1995 Physica Scripta 52184

[79] Lozovik Y E, Tsvetus V G, and Vinogradov E A 1995 JETP Lett. 61723

[80] Yablonovitch E 1989 Phys. Rev. Lett. 621742

[81] Crocce M, D. A. R. Dalvit D A R, and Mazzitelli F D 2001 Phys. Rev. A 64013808

[82] Dodonov V V 2005, talk at the Seventh International Workshop on Quantum Field Theory under the Influence of External Conditions, Barcelona

[83] Braggio C et al. 2004 Rev. Sci. Instr. 754967

[84] Braggio C et al. 2005 Europhys. Lett. 70754

[85] Braggio C et al. 2005 Optics Express 135302

[86] Ruby R, Bradley P, Larson III J D, and Oshmyansky Y 1999 Electron. Lett. 35794

[87] Zhang T et al. 2005 Chin. Phys. Lett. 22694

[88] Dodonov V V and Klimov A B 1996 Phys. Rev. A 532664

[89] Dodonov V V 1998 Phys. Rev A 584147

[90] Goy P et al. 1982 Phys. Rev. A 262065

[91] Gallagher T F 1994 Rydberg Atoms (New York: Cambridge University Press)

[92] Hulet R and Kleppner D 1983 Phys. Rev. Lett. 511430

[93] Raimond J M, M. Brune M, and S. Haroche S 2001, Rev. Mod. Phys. 73565

[94] Jaksch D et al. 2000, Phys. Rev. Lett. 852208

[95] Yamada S et al. 2005 Phys. Rev. A 72033414

[96] Haseyama T et al. 2003 Phys. Lett. A 317450

[97] Goy P et al. 1982 Phys. Rev. A 262065

[98] Moi L et al. 1983 Phys. Rev. A 272043 